\title{
Status of maize stalk rot complex in western belts of Nepal and its integrated management
}

\author{
${ }^{1}$ Subash Subedi*, ${ }^{2}$ Himalaya Subedi and ${ }^{1}$ Sarswati Neupane \\ ${ }^{1}$ Nepal Agricultural Research Council, \\ National Maize Research Program, Rampur, Chitwan, Nepal \\ ${ }^{2}$ CIMMYT-Nepal, Dang \\ *Corresponding author email: subedi.subash1@gmail.com
}

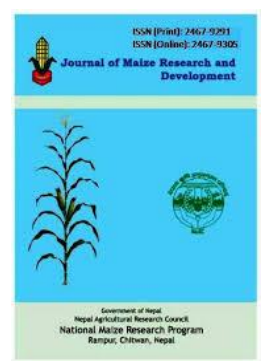

Received: September 2016; Revised: October 2016; Accepted: November 2016

\begin{abstract}
Maize stalk rot complex is becoming a serious threat for maize growing areas of Nepal. A field monitoring for maize stalk rot complex was done during crop season (August, 2016) covering 10 farmers field each of Surkhet, Banke, Dang, Chitwan and Nawalparasi districts. Maize crop showed highly susceptible reaction to the disease at western belts of Dang and susceptible reaction was marked in Chitwan and Nawalparasi districts while the disease effect was mild at Banke and Surkhet district. Most of the plant diseases managed successfully through the application of biocontrol agents, host resistance, chemicals and other different cultural control methods. The result of field experiment conducted at Dang showed that all the treatments had significant $(\mathrm{P} \leq 0.05)$ effect on percent disease index (PDI) and crop yield over farmers practice to control maize stalk rot. The higher percent disease control $(52.36 \%)$ and yield increase $(40.29 \%)$ were recorded from the plot sprayed with streptocyclin@ $2 \mathrm{~g} \mathrm{~L}^{-1}$ and insecticide (cypermethrin + chloropyrifos @ $2.5 \mathrm{ml} \mathrm{L}^{-1}$ of water during knee height and subsequent spray after 15 days interval as compared to farmers practice. Out of 30 genotypes, Rampur composit, Arun 2, Rampur 34, RamS03F08, TLBRS07F16 and Rampur 24 were found resistant against stalk rot complex with higher yield at Rampur Chitwan.
\end{abstract}

Keywords: Disease management, Stalk rot, Maize

Correct citation: Subedi, S., Subedi, H., \& Neupane. S. (2016). Status of maize stalk rot complex in western belts of Nepal and its integrated management. Journal of Maize Research and Development, 2(1): 30-42, doi: http://dx.doi.org/10.3126/jmrd.v2i1.16213

\section{INTRODUCTION}

Stalk rot complex of maize is now recognized as a remarkable problem in tropical and subtropical maize growing areas of Nepal. Usually post flowering maize stalk rot is prominent than pre-flowering to reduce maize yield. The pre-flowering types of stalk rot includes pythium stalk rot (Pythium aphanidermatum) and bacterial stalk rot (Erwinia chrysanthemi pv. Zeae), whereas others, such as Fusarium wilt, late wilt (Cephalosporium maydis), black bundle disease and charcoal rot (Macrophomina phaseolina), appear in the post-flowering phase (Subedi, 2015). Stalk rot is distributed throughout the country, but it is most prevalent in the hot and humid areas 
like Dang, Chitwan, Nawalparasi and Surkhet however Pythium stalk rot is found to be common in the mountains and the valleys in Nepal (Diwakar \& Payak, 1975). From global point of view, an estimated yield loss of 9-10\% have been reported due to stalk rot complex and which varied 4\% in northern Europe and $14 \%$ in South Asia and West Africa (Oerke, 2005). In Nepal bacterial stalk rot of maize (Erwinia chrysanthemi pv Zeae) can cause up to $80 \%$ yield loss along with other fungal diseases in the terai area (Burlakoti \& KC, 2004). Although several works have been done to cope up with other maize diseases but research activities were less in the maize stalk rot complex management in Nepal. Therefore, an instant effort is needed to manage stalk rot for tropical and subtropical maize growers. Another bitter fact is that stalk rot complex slowly becomes a serious threat in most of the terai and mid hill-low lying maize growing areas of Nepal. The complete package including development of disease resistant / tolerant variety with management practices would be effective to maize growers to tackle with the biotic constraints they faced and ultimately help to increase the maize productivity too.

\section{MATERIALS AND METHODS}

\section{Disease monitoring}

A field monitoring for stalk rot complex of maize was done during crop season (August 2016) covering about 10 farmers field each of 5 potential maize growing districts - Chitwan, Nawalparsi, Dang, Banke and Surkhet. For the surveillance, concerned officers from the respective DADOs and scientists from the NMRP, NARC research stations and CIMMYT were involved. The disease data were recorded from 10 randomly tagged plants/plot on the basis of 1-9 scoring scale (ICAR, 2012).

1 - Healthy or slight discoloration at the site of inoculation

2 - $\quad$ Up to $50 \%$ of the inoculated inter-node is discolored.

3 - $\quad 51-75 \%$ of the inoculated inter-node is discolored.

4 - $\quad 76-100 \%$ of the inoculated inter-node is discolored.

5 - $\quad$ Less than $50 \%$ discoloration of the adjacent inter-node.

6 - $\quad$ More than $50 \%$ discoloration of the adjacent inter-node.

7 - Discoloration of three internodes.

8 - Discoloration of four internodes.

9 - Discoloration of five or more internodes and premature death of plant

Based on the counts, disease incidence and index (severity) were recorded and suspected diseased specimens were collected for isolation and identification of pathogens under laboratory condition. Disease incidence and Percent Disease Index (PDI) (Wheeler, 1969) were calculated based on the following formula;

$$
\text { Disease incidence }(\%)=\frac{\text { No. of infected plants }}{\text { Total no of plant assessed }} \times 100
$$

$$
\text { PDI }(\%)=\frac{\text { Sum of all numerical values }}{\text { No of plants observed }} \times \frac{100}{\text { Maximum diseases rating }}
$$


A disease monitoring form was developed to record the surveyor name, location detail, date of the survey, latitude, longitude and elevation of the survey site, crop growth stage, field area size, if disease sample collected (sample ID number) and finally any comments or observations to understand the socio economic impact of the disease. Disease maps were developed by using disease data of surveyed area.

\section{Screening of host resistance}

Thirty genotypes were tested for maize stalk rot resistance during summer season of 2016 in NMRP, Rampur. The experiment was carried out at natural epiphytotic condition following randomized complete block design with 2 replications. The plot size was $5 \mathrm{~m}$ long with $75 \mathrm{~cm}$ row to row spacing and each genotype was sown in two rows. Agronomic practices were followed as recommended. The recommended fertilizers @ 120:60:40 $\mathrm{kg} \mathrm{ha}^{-1}$ (N:P:K) were applied. Early plant stand, tasseling days, silking days, plant height, ear height and final plant stand were recorded. The disease severity data were recorded thrice at an interval of 10 days as described in earlier activity. The area under disease progress curve (AUDPC) was computed using midpoint rule method (Campbell \& Madden, 1990). The yield data $\left(\mathrm{kg} \mathrm{ha}^{-1}\right)$ and Thousand seed weight (g) were recorded.

\section{Integrated management experiment}

The experiment was conducted under natural epiphytotic condition following Randomized Complete Block design in 3 farmer's field of Pabannagar, Dang valley and considered as a hot spot for maize stalk rot severity. The unit plot size was six rows 5 mmeter long with $75 \mathrm{~cm}$ row to row spacing i.e. $22.5 \mathrm{~m}^{2}$ gross plot area. A maize variety Rampur Composit was sown on May $28^{\text {th }}$ of 2016 in all 3 fields. There were eight treatments of the experiment including cultural, agronomical, biological and chemical practices and compared with farmers practice. The treatments combinations for the experiment were designed as follows.

1. Bavistin seed treatment @ $2 \mathrm{~g} \mathrm{~kg}^{-1}$ of seed + Saafulizer $(2.5 \mathrm{~g} \mathrm{SAAF}+300 \mathrm{~g}$ Urea) during knee height and tasseling stage

2. Basal Application of high dose of phosphorous $\left(80 \mathrm{~kg} \mathrm{ha}^{-1}\right)$ and potassium $\left(60 \mathrm{~kg} \mathrm{ha}^{-1}\right)$

3. Spray streptocyclin@2 $\mathrm{g} \mathrm{L}^{-1}+$ insecticide (cypermethrin + chloropyrifos) @ $2.5 \mathrm{ml} / \mathrm{l}$ of water during knee height and subsequent spray at 15 days interval

4. Seed treatment with Trichoderma viridae @ (One vial of $5 \mathrm{ml}\left(1 \times 10^{8}\right.$ conidia/ml) $/ \mathrm{kg}$ of seed) + soil application @ (One vial of $5 \mathrm{ml}\left(1 \times 10^{8}\right.$ conidia/ml $) / 10 \mathrm{~kg}$ of FYM) per plot mixed during field preparation

5. Seed treatment with Pseudomonas fluorescence @ (One vial of $5 \mathrm{ml}\left(1 \times 10^{8}\right.$ spore $\left./ \mathrm{ml}\right) / \mathrm{kg}$ of seed) + soil application @ (One vial of $5 \mathrm{ml}\left(1 \times 10^{8}\right.$ spore $\left./ \mathrm{ml}\right) / 10 \mathrm{~kg}$ of FYM) per plot mixed during field preparation and vegetative stage both

6. Earthing up with appropriate plant population $(75 \times 25 \mathrm{~cm}$ spacing $)$ for well drainage of excess water

7. Intercropping of maize with soybean (1:2 ratio) in raised bed system + copper oxychloride @ $2 \mathrm{~g} / \mathrm{l}$ of water during knee height and subsequent spray after 15 days interval

8. Farmers practice (Control). 
All treatments were replicated three times. One farmer was considered as one replication. In case of chemical, first spray was given during knee height stage and another after 15 days interval of first spray. Disease severity data was recorded before every treatment application using 1-9 scoring scale from 25 randomly tagged plants/plot as described in earlier activity. The AUDPC and PDI were calculated as described in earlier activity. Percent disease control (PDC) was calculated on the basis of the formula developed by Shivankar and Wangikar (1993). Early Plant Stand (EPS) and Final Plant Stand (FPS) were recorded as described earlier. Data was recorded on yield $\left(\mathrm{kg} \mathrm{ha}^{-1}\right)$ and yield attributes after necessary sun drying. Yield increase over the farmer practice was calculated. All data were analyzed statistically using Microsoft Excel and MSTAT-C computer package program. Treatment means were compared using Duncan's Multiple Range Test (DMRT) at 5\% levels of significance. All percent data were subjected to arcsine transformation before statistical analysis. Disease maps were developed by using ArcGIS 10.3 software.

\section{Disease monitoring}

\section{RESULTS AND DISCUSSIONS}

The stalk rot complex symptoms were found very common and damaging in maize fields at western belts of Dang (80.86\% PDI and 65.00\% incidence), Chitwan (61.82\% PDI and 61.00\% incidence) and Nawalparasi (55.55\% PDI and $52.00 \%$ incidence) respectively (Table 1)

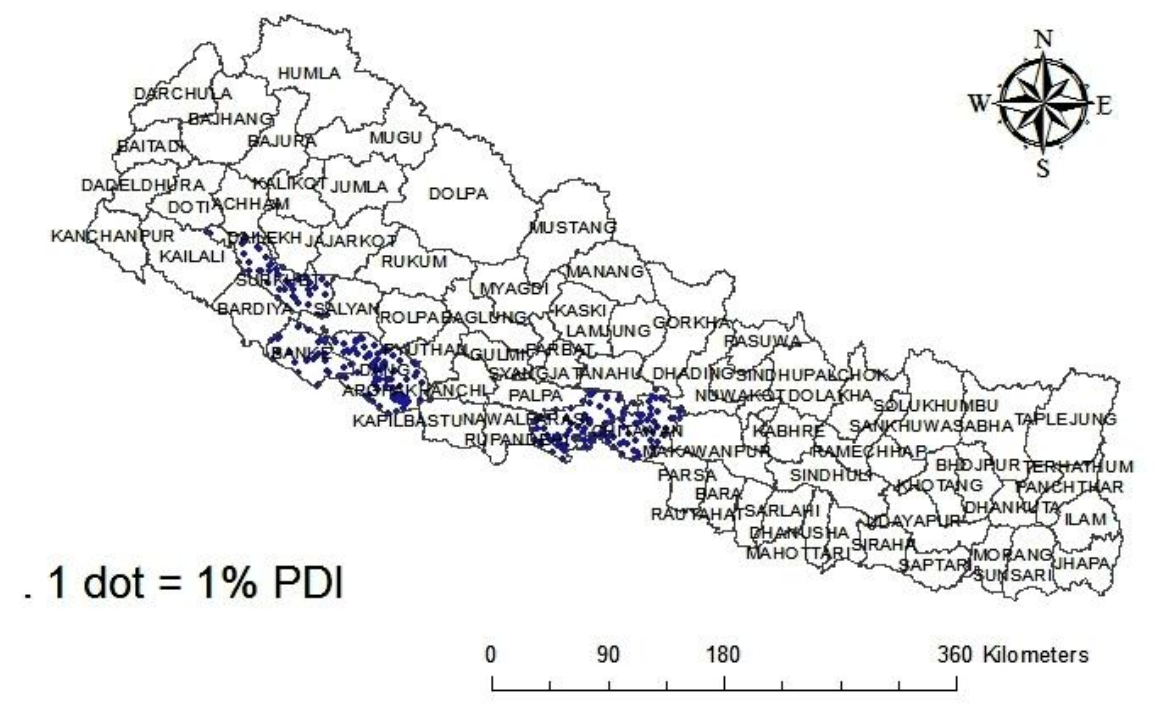

Figure 1. A disease map for disease index (Severity) of maize stalk rot complex at 5 maize growing districts of Nepal monitored during summer season (2016).

The lower disease index of $23.52 \%$ with $14.00 \%$ incidence was recorded in Khaskusum area of Banke district followed by Surkhet having 43.57\% PDI and $29.00 \%$ incidence where crop showed resistant to moderately susceptible reaction to the disease (Table 1). Disease maps for disease index or severity (Figure 1) and disease incidence (Figure 2) were developed by using the 
Journal of Maize Research and Development (2016) 2 (1): 30-42

ISSN: 2467-9291 (Print), 2467-9305 (Online)

DOI: http://dx.doi.org/10.3126/jmrd.v2i1.16213

disease data of PDI and incidence of 5 monitored districts during August 2016 where 1 dot represents 1\% PDI for Figure 1 and 1\% incidence for Figure 2. Districts with higher dot densities were considered as severely infected districts for maize stalk rot complex while districts with lower dot densities were showed mild response to the disease severity and incidence.

Table 1. Disease index and incidence of maize stalk rot complex in 5 maize growing districts of Nepal during summer season of 2016

\begin{tabular}{|c|c|c|c|c|c|c|c|}
\hline \multirow[t]{3}{*}{ District /VDCs } & \multicolumn{6}{|c|}{ Year (2016) } & \multirow{3}{*}{$\begin{array}{l}\text { Disease } \\
\text { reaction }\end{array}$} \\
\hline & \multicolumn{3}{|c|}{$\begin{array}{l}\text { Disease index (PDI) } \\
(\mathrm{n}=10)\end{array}$} & \multicolumn{3}{|c|}{ Disease incidence $(\mathrm{n}=10)$} & \\
\hline & Mean & SD & $\mathrm{SE} \pm$ & Mean & SD & $\mathrm{SE} \pm$ & \\
\hline Banke (Khaskusum) & 23.52 & 5.51 & 1.74 & 14.00 & 5.16 & 1.63 & $\mathrm{R}$ \\
\hline Surkhet (Dasarathpur and Mayalkuna) & 43.57 & 5.29 & 1.67 & 29.00 & 7.38 & 2.33 & MS \\
\hline Dang (Aswara and Panchakule) & 80.86 & 11.00 & 3.48 & 65.00 & 12.69 & 4.01 & HS \\
\hline Chitwan (Mangalpur) & 61.82 & 8.33 & 2.63 & 61.00 & 8.76 & 2.77 & S \\
\hline Nawalparasi (Gaindakot and Rajahar) & 55.55 & 10.55 & 3.34 & 52.00 & 12.29 & 3.89 & S \\
\hline
\end{tabular}

Note : PDI- Percent Disease Index, n10 = sample size 10, SD- Standard deviation, SE- Standard error, HS- Highly susceptible, SSusceptible, R-Resistant, MS- Moderately Susceptible

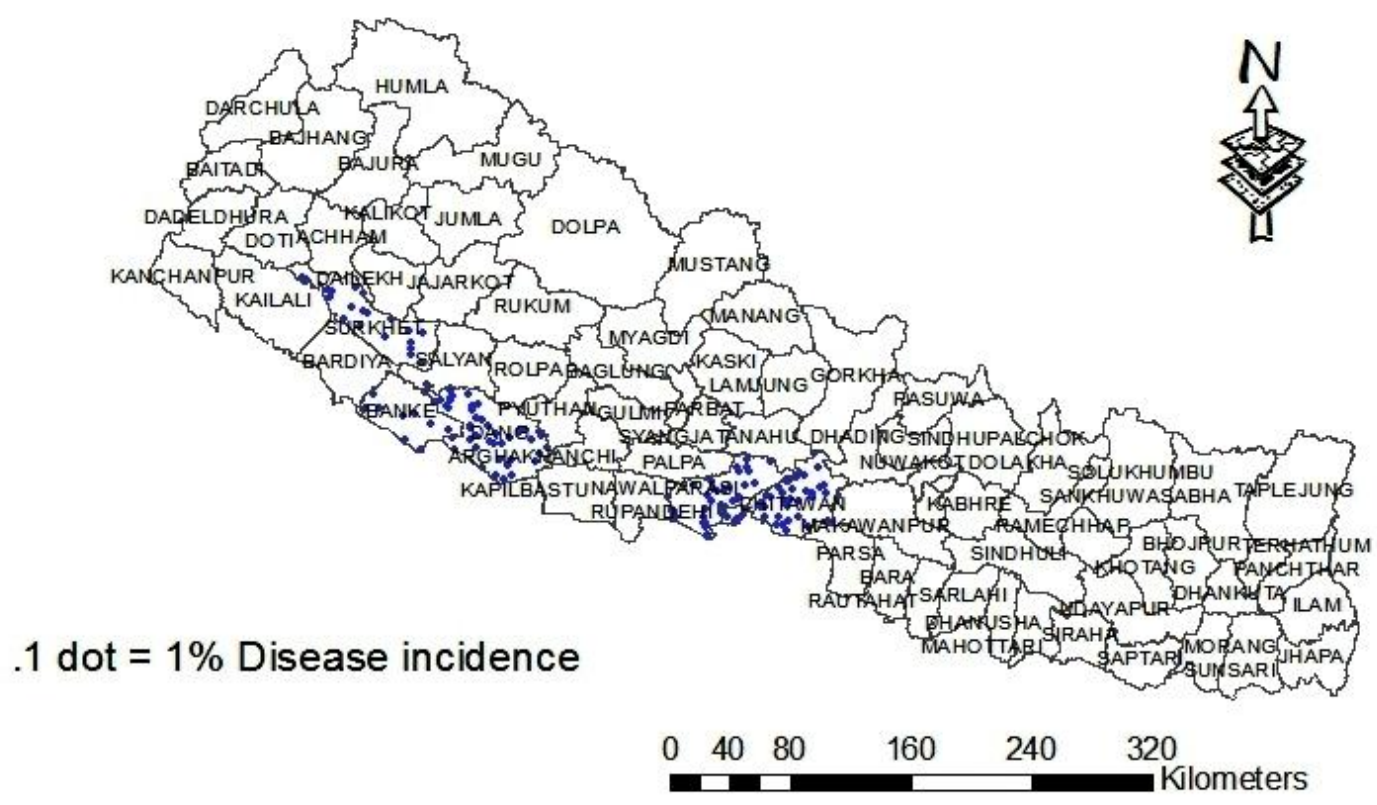

Figure 2. A disease map for disease incidence of maize stalk rot complex at 5 maize growing districts of Nepal monitored during summer season of 2016.

\section{Screening of host resistance}

The summer maize season of 2016 was affable for stalk rot complex development. The early plant stand, disease severity, final plant stand, grain yield and thousand seed weight were significantly $(\mathrm{P} \leq 0.05)$ varied among the tested maize genotypes during experiment period of 2016 at Rampur, Chitwan. 
Journal of Maize Research and Development (2016) 2 (1): 30-42

ISSN: 2467-9291 (Print), 2467-9305 (Online)

DOI: http://dx.doi.org/10.3126/jmrd.v2i1.16213

Table 2. Screening of maize genotypes for stalk rot complex resistance at Rampur, Chitwan during 2016.

\begin{tabular}{|c|c|c|c|c|c|c|c|c|}
\hline \multirow[t]{2}{*}{ Genotypes } & \multirow[t]{2}{*}{ EPS } & \multicolumn{3}{|c|}{ Disease Severity (1-9) } & \multirow[t]{2}{*}{ AUDPC } & \multirow[t]{2}{*}{ FPS } & \multirow{2}{*}{$\begin{array}{l}\text { GY } \\
\left(\mathrm{kg} \mathrm{ha}^{-1}\right)\end{array}$} & \multirow[t]{2}{*}{ TSWt $(\mathrm{g})$} \\
\hline & & 60 DAS & 70 DAS & 80 DAS & & & & \\
\hline Rampur Composit & $34.50^{\dagger}$ & 1.53 & 2.30 & 4.53 & 53.25 & 29.50 & 2126.52 & 315.00 \\
\hline Arun 2 & 36.50 & 1.78 & 2.40 & 4.70 & 56.38 & 29.00 & 1987.18 & 347.50 \\
\hline Poshilo Makai 1 & 30.00 & 3.15 & 3.60 & 6.05 & 82.00 & 13.50 & 1343.97 & 282.50 \\
\hline S99TLYQ-B & 37.00 & 3.15 & 3.58 & 6.05 & 81.75 & 22.00 & 1328.66 & 285.00 \\
\hline S99TLYQ-HG-AB & 39.50 & 3.98 & 4.48 & 6.88 & 99.00 & 18.00 & 834.68 & 315.00 \\
\hline BGBYPOP & 35.00 & 2.80 & 3.30 & 5.70 & 75.50 & 20.50 & 1539.79 & 205.00 \\
\hline R pop-3 & 33.50 & 3.28 & 3.78 & 6.30 & 85.63 & 16.50 & 1231.59 & 335.00 \\
\hline R pop-4 & 37.00 & 2.60 & 2.98 & 5.40 & 69.75 & 26.50 & 1643.21 & 267.50 \\
\hline Rampur Hybrid 4 & 35.00 & 2.63 & 2.99 & 5.44 & 70.20 & 20.50 & 1626.38 & 350.00 \\
\hline Rampur Hybrid 6 & 34.00 & 2.88 & 3.40 & 5.80 & 77.38 & 18.50 & 1509.41 & 220.00 \\
\hline RML 95/RML 96 & 39.50 & 4.20 & 4.70 & 7.08 & 103.38 & 18.00 & 864.42 & 275.00 \\
\hline RAMS03F08 & 33.50 & 2.20 & 2.70 & 4.95 & 62.75 & 26.50 & 1948.57 & 272.50 \\
\hline $\mathrm{ZM} 401$ & 32.50 & 2.69 & 3.08 & 5.60 & 72.20 & 19.50 & 1669.61 & 342.50 \\
\hline ZM 627 & 42.00 & 5.08 & 5.30 & 7.40 & 115.38 & 16.50 & 504.51 & 387.50 \\
\hline 05 SADVI & 34.00 & 3.80 & 4.28 & 6.63 & 94.88 & 14.50 & 1034.42 & 260.00 \\
\hline 07 SADVI & 35.00 & 3.50 & 4.00 & 6.43 & 89.63 & 16.00 & 1123.63 & 335.00 \\
\hline Rampur 21 & 38.00 & 5.33 & 5.80 & 8.08 & 125.00 & 11.00 & 332.97 & 362.50 \\
\hline Rampur 24 & 34.00 & 3.70 & 4.20 & 6.63 & 93.63 & 15.00 & 1033.14 & 305.00 \\
\hline Rampur 27 & 36.00 & 2.43 & 2.95 & 5.20 & 67.63 & 25.00 & 1731.09 & 357.50 \\
\hline Rampur 32 & 27.50 & 2.80 & 3.20 & 5.65 & 74.25 & 12.50 & 1660.09 & 352.50 \\
\hline Rampur 33 & 33.00 & 3.60 & 4.08 & 6.55 & 91.50 & 14.00 & 1047.43 & 267.50 \\
\hline Rampur 34 & 32.50 & 1.98 & 2.60 & 4.90 & 60.38 & 24.50 & 1935.05 & 367.50 \\
\hline Rampur 36 & 37.00 & 3.08 & 3.58 & 6.05 & 81.38 & 20.50 & 1363.18 & 275.00 \\
\hline TLBRS07F16 & 37.50 & 2.40 & 2.90 & 5.08 & 66.38 & 25.50 & 1762.28 & 415.00 \\
\hline Across $9331 \mathrm{RE}$ & 35.50 & 3.40 & 3.90 & 6.33 & 87.63 & 19.50 & 1181.24 & 365.00 \\
\hline Across 9942/Ac 9944 & 29.50 & 3.73 & 4.30 & 6.68 & 95.00 & 8.00 & 1032.72 & 352.50 \\
\hline BLBSRS07F10 & 37.00 & 2.93 & 3.43 & 5.88 & 78.25 & 23.00 & 1540.43 & 317.50 \\
\hline TLBRS07F14 & 36.00 & 3.63 & 4.13 & 6.65 & 92.63 & 17.50 & 1075.79 & 357.50 \\
\hline Arun-4 & 40.00 & 2.84 & 3.28 & 5.73 & 75.58 & 26.00 & 1545.13 & 315.00 \\
\hline Farmer's Local (SC) & 41.00 & 5.20 & 5.55 & 7.58 & 119.38 & 14.00 & 421.61 & 315.00 \\
\hline Grand mean & 35.43 & 3.21 & 3.69 & 6.06 & 83.25 & 19.38 & 1332.62 & 317.33 \\
\hline F-test & $* *$ & $* *$ & $* *$ & $* *$ & $* *$ & $* *$ & $* *$ & $* *$ \\
\hline $\operatorname{LSD}(\leq 0.05)$ & 2.89 & 0.08 & 0.10 & 0.14 & 1.82 & 1.79 & 65.15 & 16.04 \\
\hline $\mathrm{CV} \%$ & 3.98 & 1.27 & 1.34 & 1.11 & 1.07 & 4.50 & 2.39 & 2.47 \\
\hline
\end{tabular}

${ }^{\dagger}$ Means of 2 replications. EPS- Early Plant Stand, AUDPC- Area under Disease Progress Curve, FPS- Final Plant Stand, GY- Grain Yield (kilogram/hectare), TSWt- Thousand Seed Weight (gram), DAS- Days after Sowing, SC-

Susceptible Check, **- highly significant 
Journal of Maize Research and Development (2016) 2 (1): 30-42

ISSN: 2467-9291 (Print), 2467-9305 (Online)

DOI: http://dx.doi.org/10.3126/jmrd.v2i1.16213

Table 3. Evaluation of agronomic traits in maize genotypes in stalk rot complex screening nursery at Rampur, Chitwan during 2016.

\begin{tabular}{|c|c|c|c|c|}
\hline Genotypes & $50 \%$ Tasseling & $50 \%$ Silking & Plant height $(\mathrm{cm})$ & Ear height $(\mathrm{cm})$ \\
\hline Rampur Composit & $48.00^{\dagger}$ & 52.00 & 180.00 & 95.50 \\
\hline Arun 2 & 46.00 & 49.50 & 180.00 & 88.00 \\
\hline Poshilo Makai 1 & 48.00 & 52.00 & 178.00 & 90.50 \\
\hline S99TLYQ-B & 48.50 & 52.00 & 156.00 & 83.00 \\
\hline S99TLYQ-HG-AB & 52.00 & 55.00 & 171.00 & 86.50 \\
\hline BGBYPOP & 47.50 & 51.50 & 164.50 & 87.00 \\
\hline $\mathrm{R}$ pop-3 & 50.00 & 52.50 & 147.00 & 88.00 \\
\hline $\mathrm{R}$ pop-4 & 48.50 & 52.00 & 176.00 & 100.00 \\
\hline Rampur Hybrid 4 & 54.50 & 57.50 & 149.00 & 73.50 \\
\hline Rampur Hybrid 6 & 54.50 & 57.50 & 160.00 & 81.00 \\
\hline RML 95/RML 96 & 56.50 & 59.50 & 174.00 & 94.50 \\
\hline RAMS03F08 & 50.50 & 54.00 & 185.00 & 99.50 \\
\hline ZM 401 & 48.50 & 52.50 & 159.50 & 83.00 \\
\hline ZM 627 & 52.50 & 55.50 & 149.50 & 74.50 \\
\hline 05 SADVI & 54.00 & 57.00 & 150.00 & 69.50 \\
\hline 07 SADVI & 53.50 & 57.50 & 166.00 & 81.50 \\
\hline Rampur 21 & 51.50 & 54.50 & 184.00 & 75.00 \\
\hline Rampur 24 & 55.00 & 58.00 & 135.50 & 60.00 \\
\hline Rampur 27 & 56.50 & 59.50 & 160.50 & 79.00 \\
\hline Rampur 32 & 55.00 & 58.00 & 172.50 & 84.50 \\
\hline Rampur 33 & 54.00 & 58.50 & 139.50 & 73.00 \\
\hline Rampur 34 & 55.00 & 59.00 & 154.00 & 65.50 \\
\hline Rampur 36 & 55.00 & 58.50 & 163.50 & 73.00 \\
\hline TLBRS07F16 & 57.50 & 60.50 & 173.00 & 87.50 \\
\hline Across $9331 \mathrm{RE}$ & 49.50 & 52.50 & 149.50 & 61.50 \\
\hline Across 9942/Ac 9944 & 54.00 & 57.50 & 171.00 & 73.00 \\
\hline BLBSRS07F10 & 50.50 & 53.50 & 174.00 & 87.00 \\
\hline TLBRS07F14 & 56.50 & 59.50 & 157.00 & 75.00 \\
\hline Arun-4 & 47.00 & 50.00 & 143.00 & 70.00 \\
\hline Farmer's Local (SC) & 57.50 & 60.50 & 172.00 & 83.50 \\
\hline Grand mean & 52.25 & 55.58 & 163.15 & 80.77 \\
\hline F-test & $* *$ & $* *$ & $* *$ & $* *$ \\
\hline $\mathrm{LSD}(\leq 0.05)$ & 4.98 & 4.91 & 7.50 & 5.42 \\
\hline $\mathrm{CV} \%$ & 4.66 & 4.32 & 2.25 & 3.28 \\
\hline
\end{tabular}

\footnotetext{
${ }^{\dagger}$ Means of 2 replication. Cm- centimeter, SC- Susceptible Check, **- highly significant
} 
Out of 30 genotypes, Rampur Composite, Arun 2, RamS03F08, Rampur 34, TLBRS07F16 and Rampur 24 were resistant having area under disease progress curve (AUDPC) value of $53.25,56.38,60.38,62.75,66.38$ and 67.6 respectively (Table 2). The other remaining genotypes showed moderately susceptible and susceptible reaction to the disease. The high yielding genotypes were Rampur Composite $\left(2126.52 \mathrm{~kg} \mathrm{ha}^{-1}\right)$, Arun-2 (1987.18 kg ha-1), RAMS03F08 (1948.57 kg ha'), Rampur 34 (1935.05 $\left.\mathrm{kg} \mathrm{ha}^{-1}\right)$, TLBRS07F16 (1762.28 kg ha $\left.{ }^{-1}\right)$ and Rampur 24 (1731.09 $\mathrm{kg} \mathrm{ha}^{-1}$ ) (Table 2). The genotypes having higher thousand seed weight were TLBRS07F16 (415 g), ZM 627 (387.5 g), Rampur 34 (367.5 g), Across 9331 RE (365 g), Rampur 21 (362.5 g) and Rampur 27 (357.5 g) (Table 2).

\section{Relationship between grain yield (kg/ha) and AUDPC}

During summer maize season (2016), among 6 (3 high yielding genotypes - Rampur Composit, Arun 2, RAMS03F08 and 3 low yielding genotypes ZM 627, Farmers local and Rampur 21), grain yield was found to had highly significant negative correlation $(\mathrm{r}=-0.99)$ with the AUDPCof maize stalk rot complex disease. The predicted linear regression line also displayed downward slope i.e. $y=-0.039 x+136.5$, with regression coefficient $R^{2}=0.99$, where ' $y$ ' denoted predicted crop yield of maize genotypes and ' $x$ ' stood for AUDPC of stalk rot complex of maize (Figure 3). The estimated regression line indicated that the unit rise in the AUDPC of stalk rot complex disease (within 1-9 scale), there existed possibilities of yield reduction by $0.039 \mathrm{~kg} \mathrm{ha}^{-1}$.

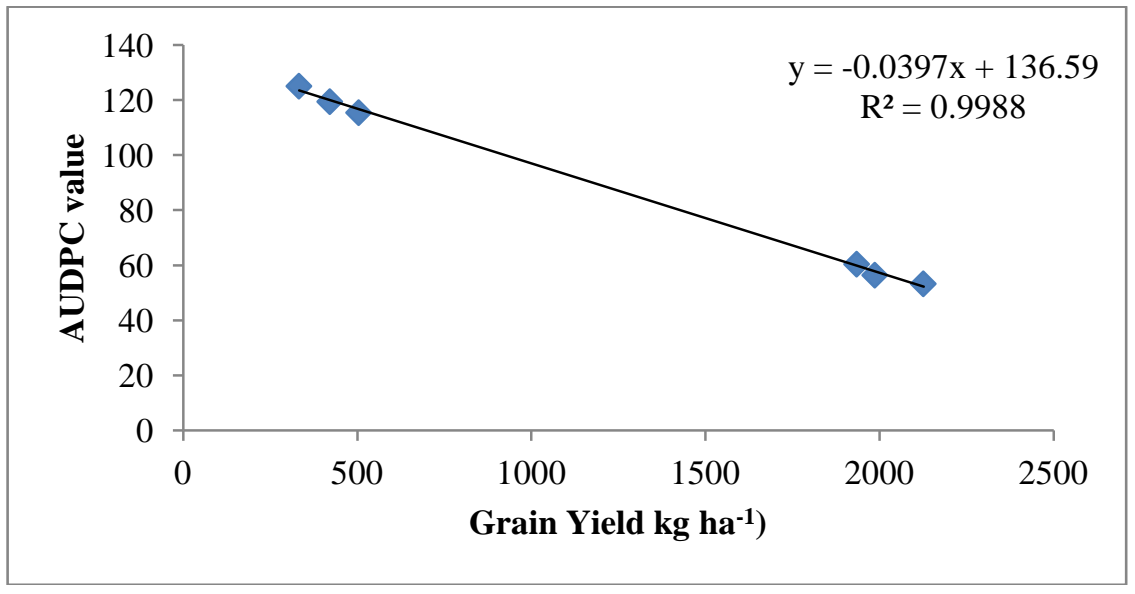

Figure 3. Relationship between crop yield ( $\mathrm{kg} / \mathrm{ha}$ ) and AUDPC of maize stalk rot complex in screening experiment at Rampur, Chitwan during 2016

The result showed that tasseling days varied from 46 days (Arun 2) to 57.5 days (TLBRS07F16). Similarly, the silking day varied from 49.5 days (Arun 2) to 60.5 days (TLBRS07F16). The tested genotypes were highly significant for tasseling and silking days (Table 3). The plant height varied from $135.50 \mathrm{~cm}$ (Rampur 24) to $185.00 \mathrm{~cm}$ (RAMS03F08). Similarly, the ear height also varied from $60.00 \mathrm{~cm}$ (Rampur 24) to $100.00 \mathrm{~cm}$ (R pop-4). The plant height and ear height were significantly varied among the tested genotypes (Table 3 ). 


\section{Integrated management}

All the treatments had significant $(\mathrm{P} \leq 0.05)$ effect on percent disease index (PDI) and crop yield over farmers practice. The higher percent disease control (52.36\%) and yield increase $(40.29 \%)$ were recorded from the plot sprayed with streptocyclin @ $2 \mathrm{~g} / \mathrm{l}$ and insecticide (cypermethrin + chloropyrifos) @ $2.5 \mathrm{ml} / 1$ of water during knee height and subsequent spray after 15 days interval as compared to farmers practice (Table 5). Similarly, the lower percent disease index (52.65\% PDI) with higher yield (3589.00 $\left.\mathrm{kg} \mathrm{ha}^{-1}\right)$ was also found in the plot where maize seed were treated with Bavistin as a seed treatment @ $2 \mathrm{~g} \mathrm{~kg}^{-1}$ of seed and soil application of Saafulizer (2.5 g SAAF + $300 \mathrm{~g}$ Urea) during knee height and tasseling stage as compared to farmer practice (PDI- 85.75\% and yield $-2760.00 \mathrm{~kg} \mathrm{ha}^{-1}$ ) (Table 4). The plot applied with basal application of high dose of phosphorous $\left(80 \mathrm{~kg} \mathrm{ha}^{-1}\right)$ and potassium $\left(60 \mathrm{~kg} \mathrm{ha}^{-1}\right)$ recorded significantly lower PDI (65.75\%) (Table 4) with higher yield increase (19.71\%) as compared to farmer practice (Table 5).

\section{Relationship between disease control and yield increase}

During the experimental period, the yield increase showed significantly highly positive correlation $(\mathrm{r}=0.99)$ with the controlled maize stalk rot complex disease by the application of cultural, biological and chemical means. The predicted linear regression line was displayed upward slope i.e. $y=0.799 x-0.463$, with regression coefficient $R^{2}=0.98$, where ' $y$ ' denoted predicted yield increase of maize and ' $x$ ' stood for disease control due to applied treatments (Figure 4). The estimated regression line indicated that the unit rise in the percent disease control of maize stalk rot complex (within 1-9 scale) due to applied treatments, there existed possibilities of yield increase by 0.80 percent.

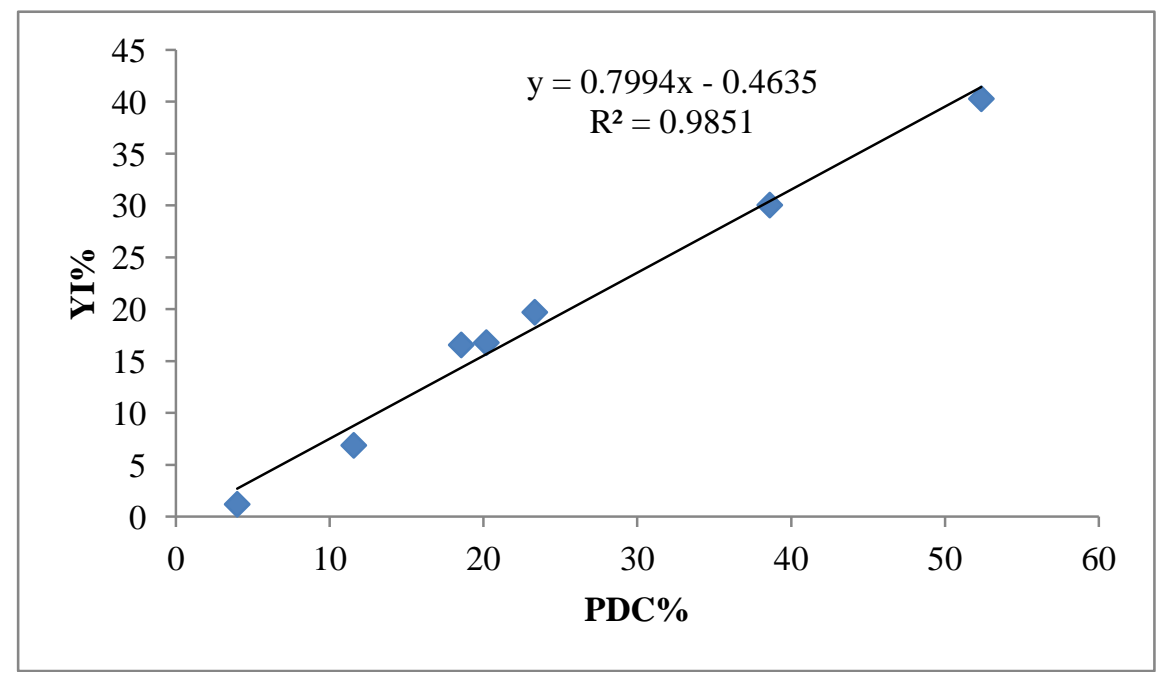

Figure 4. Relationship between disease control and yield increase in disease management experiment through cultural, biological and chemical means at Pabannagar, Dang during 2016. 
Journal of Maize Research and Development (2016) 2 (1): 30-42

ISSN: 2467-9291 (Print), 2467-9305 (Online)

DOI: http://dx.doi.org/10.3126/jmrd.v2i1.16213

Table 4. Effect of cultural, biological and chemical practices on stalk rot complex severity and yield performance of maize at Pabannagar, Dang during 2016.

\begin{tabular}{|c|c|c|c|c|c|c|}
\hline Treatments & EPS & AUDPC & PDI\% & FPS & $\begin{array}{c}\text { Yield } \\
\left(\mathrm{kg} \mathrm{ha}^{-1}\right)\end{array}$ & $\begin{array}{l}\text { TSWt } \\
(\mathrm{g})\end{array}$ \\
\hline $\begin{array}{l}\text { 1. Bavistin seed treatment @ } 2 \mathrm{~g} \mathrm{~kg}^{-1} \text { of seed }+ \\
\text { Saafulizer }(2.5 \mathrm{~g} \mathrm{SAAF}+300 \mathrm{~g} \text { Urea }) \text { during knee } \\
\text { height and tasseling stage }\end{array}$ & $126.67^{\dagger}$ & $49.70^{g}$ & $52.65^{\mathrm{g}}$ & $102.30^{\mathrm{ab}}$ & $3589.00^{\mathrm{b}}$ & $370.70^{\mathrm{a}}$ \\
\hline $\begin{array}{l}\text { 2. Basal Application of high dose of phosphorous } \\
(80 \mathrm{~kg} / \mathrm{ha}) \text { and potassium }\left(60 \mathrm{~kg} \mathrm{ha}^{-1}\right) \text {. }\end{array}$ & 122.33 & $69.08^{f}$ & $65.75^{\mathrm{f}}$ & $98.67^{\mathrm{bc}}$ & $3304.00^{\mathrm{c}}$ & $355.00^{\mathrm{b}}$ \\
\hline $\begin{array}{l}\text { 3. Spray streptocyclin @2 } \mathrm{g} \mathrm{L}^{-1}+\text { insecticide } \\
\text { (cypermethrin + chloropyrifos @ } 2.5 \mathrm{ml} \mathrm{L} \mathrm{L}^{-1} \text { of } \\
\text { water during knee height and subsequent spray } \\
\text { after } 15 \text { days interval }\end{array}$ & 117.67 & $47.08^{\mathrm{h}}$ & $40.85^{\mathrm{h}}$ & $106.00^{\mathrm{a}}$ & $3872.00^{\mathrm{a}}$ & $375.70^{\mathrm{a}}$ \\
\hline $\begin{array}{l}\text { 4. Seed treatment with Trichoderma viridae @ } \\
\left.\text { (One vial of } 5 \mathrm{ml}\left(1 \times 10^{8} \text { conidia } / \mathrm{ml}\right) / \mathrm{kg} \text { of seed }\right)+ \\
\text { soil application @ (One vial of } 5 \mathrm{ml}\left(1 \times 10^{8}\right. \\
\text { conidia } / \mathrm{ml}) / 10 \mathrm{~kg} \text { of } \mathrm{FYM}) \text { per plot mixed during } \\
\text { field preparation }\end{array}$ & 127.33 & $71.83^{\mathrm{e}}$ & $68.45^{\mathrm{e}}$ & $95.67^{\mathrm{bcd}}$ & $3223.00^{\mathrm{d}}$ & $342.30^{c}$ \\
\hline $\begin{array}{l}\text { 5. Seed treatment with Pseudomonas fluorescence } \\
\left.\text { @ (One vial of } 5 \mathrm{ml}\left(1 \times 10^{8} \text { spore } / \mathrm{ml}\right) / \mathrm{kg} \text { of seed }\right)+ \\
\text { soil application @ (One vial of } 5 \mathrm{ml}\left(1 \times 10^{8}\right. \\
\text { spore } / \mathrm{ml}) / 10 \mathrm{~kg} \text { of } \mathrm{FYM}) \text { per plot mixed during } \\
\text { field preparation and vegetative stage both }\end{array}$ & 116.00 & $73.83^{\mathrm{d}}$ & $69.85^{\mathrm{d}}$ & $95.00^{\mathrm{cd}}$ & $3217.00^{\mathrm{d}}$ & $330.00^{\mathrm{d}}$ \\
\hline $\begin{array}{l}\text { 6. Earthing up with appropriate plant population } \\
(75 \times 25 \mathrm{~cm} \text { spacing }) \text { for well drainage of excess } \\
\text { water }\end{array}$ & 109.67 & $78.42^{\mathrm{c}}$ & $75.85^{\mathrm{c}}$ & $93.67^{\mathrm{cd}}$ & $2950.00^{\mathrm{e}}$ & $316.00^{\mathrm{e}}$ \\
\hline $\begin{array}{l}\text { 7. Intercropping of maize with soybean ( } 1: 2 \text { ratio) } \\
\text { in raised bed system + copper oxychloride @ } 2 \\
\mathrm{~g} \mathrm{~L}^{-1} \text { of water during knee height and subsequent } \\
\text { spray after } 15 \text { days interval }\end{array}$ & 113.67 & $82.25^{\mathrm{b}}$ & $82.35^{\mathrm{b}}$ & $93.00^{\mathrm{cd}}$ & $2793.00^{f}$ & $310.00^{\mathrm{e}}$ \\
\hline 8. Farmers practice (Control) & 107.33 & $95.25^{\mathrm{a}}$ & $85.75^{\mathrm{a}}$ & $88.67^{\mathrm{d}}$ & $2760.00^{\mathrm{f}}$ & $307.70^{\mathrm{e}}$ \\
\hline Grand mean & 117.58 & 70.93 & 67.69 & 96.63 & 3213.65 & 338.42 \\
\hline F-test & NS & $* *$ & $* *$ & $*$ & $* *$ & $* *$ \\
\hline $\operatorname{LSD}(\leq 0.05)$ & 22.99 & 1.15 & 1.13 & 6.68 & 79.28 & 9.12 \\
\hline CV\% & 11.17 & 0.93 & 0.95 & 3.95 & 1.41 & 1.54 \\
\hline
\end{tabular}

${ }^{\dagger}$ Means of 3 replication. Means in column with same superscript is not significantly different by DMRT $(\mathrm{P}<0.05)$. EPS-early plant stand, AUDPC- Area under disease progress curve, PDI-percent disease index, FPS-final plant stand, TSWt-thousand seed weight, $\mathrm{Kg} / \mathrm{ha}$ Kilogram per hectare, g- gram, \%- percent, ml-milliliter, l-liter, cm-centimeter, NS-not significant, *-significant, ${ }^{* *}$ - highly significant

Stalk rot is widespread throughout the country, but most common in the hot and humid areas (Shah, 1968). The disease usually appears at the tasseling stage (Diwakar \& Payak, 1975). The incidence of disease is significantly influenced by both environmental and host factors. The symptoms become evident after flowering and towards maturity, when plants show premature drying. The pathogen commonly affects the roots, crown region and lower internodes. When split open, the stalks show a pink-purple discoloration with collapse of the pith region (De Leon, 1984). Temperature and relative humidity have been found to affect both the growth of the pathogen and disease development. 
Journal of Maize Research and Development (2016) 2 (1): 30-42

ISSN: 2467-9291 (Print), 2467-9305 (Online)

DOI: http://dx.doi.org/10.3126/jmrd.v2i1.16213

Table 5.Effect of different treatments on stalk rot disease control and yield increase percent of maize at Pabannagar, Dang during 2016

\begin{tabular}{|c|c|c|}
\hline Treatments & PDC\% & YI\% \\
\hline $\begin{array}{l}\text { 1. Bavistin seed treatment @ } 2 \mathrm{~g} \mathrm{~kg}^{-1} \text { of seed + Saafulizer }(2.5 \mathrm{~g} \mathrm{SAAF}+ \\
300 \mathrm{~g} \text { Urea) during knee height and tasseling stage }\end{array}$ & 38.60 & 30.04 \\
\hline $\begin{array}{l}\text { 2. Basal Application of high dose of phosphorous }(80 \mathrm{~kg} / \mathrm{ha}) \text { and } \\
\text { potassium }\left(60 \mathrm{~kg} \mathrm{ha}^{-1}\right) \text {. }\end{array}$ & 23.32 & 19.71 \\
\hline $\begin{array}{l}\text { 3. Spray streptocyclin @ } 2 \mathrm{~g} \mathrm{~L}^{-1}+\text { insecticide (cypermethrin }+ \\
\text { chloropyrifos @ } 2.5 \mathrm{ml} \mathrm{L}^{-1} \text { of water during knee height and subsequent } \\
\text { spray after } 15 \text { days interval }\end{array}$ & 52.36 & 40.29 \\
\hline $\begin{array}{l}\text { 4. Seed treatment with Trichoderma viridae @ (One vial of } 5 \mathrm{ml}\left(1 \times 10^{8}\right. \\
\text { conidia/ml) } / \mathrm{kg} \text { of seed) + soil application @ (One vial of } 5 \mathrm{ml}\left(1 \times 10^{8}\right. \\
\text { conidia } / \mathrm{ml}) / 10 \mathrm{~kg} \text { of FYM) per plot mixed during field preparation }\end{array}$ & 20.17 & 16.78 \\
\hline $\begin{array}{l}\text { 5. Seed treatment with Pseudomonas fluorescence @ (One vial of } 5 \mathrm{ml} \\
\left(1 \times 10^{8} \text { spore } / \mathrm{ml}\right) / \mathrm{kg} \text { of seed) }+ \text { soil application @ (One vial of } 5 \mathrm{ml}\left(1 \times 10^{8}\right. \\
\text { spore } / \mathrm{ml}) / 10 \mathrm{~kg} \text { of FYM }) \text { per plot mixed during field preparation and } \\
\text { vegetative stage both }\end{array}$ & 18.54 & 16.56 \\
\hline $\begin{array}{l}\text { 6. Earthing up with appropriate plant population }(75 \times 25 \mathrm{~cm} \text { spacing) for } \\
\text { well drainage of excess water }\end{array}$ & 11.55 & 6.88 \\
\hline $\begin{array}{l}\text { 7. Intercropping of maize with soybean }(1: 2 \text { ratio) in raised bed system }+ \\
\text { copper oxychloride @ } 2 \mathrm{~g} \mathrm{~L}^{-1} \text { of water during knee height and subsequent } \\
\text { spray after } 15 \text { days interval } \\
\text { 8. Farmers practice (Control) }\end{array}$ & 3.97 & 1.20 \\
\hline
\end{tabular}

The maximum disease development occurs within a temperature range of $30-35^{\circ} \mathrm{C}$, with a relative humidity of $80-100 \%$ (Subedi, 2015). Waterlogged, low-lying or poorly drained field conditions favor a high degree of disease development. Plant age (pre-flowering growth stage) and a large plant population ( $\geq 60000$ per ha) favor a high incidence of disease (Diwakar and Payak, 1980). Some resistant material has been identified. Resistance to stalk rot disease involves several traits including physiological, morphological and functional characters. Maize stalk strength is determined by both stalk morphology and abiotic stress factor (Singh et al, 2012). Stalk rot infectivity depends on environmental factors, the genotype and environment interaction (G×E) and host resistance of maize genotypes to the pathogens (Szoke et al, 2007). Ledencan et al (2003) marked low disease scoring of hybrids than inbreds and differed significantly in resistance and infection types. Hybrids Ganga Safed-2, Hi-starch, and composites Suwan 1 and Suwan 2, have shown resistance in India. The findings of this experiment are also supported by Thind et al. (1984) who found that spray and soil drenching of streptocycline (100 $\mu \mathrm{g} / \mathrm{ml})$ alone and in combination with Blitox $50 \mathrm{WP}\left(2000 \mu \mathrm{g} \mathrm{ml}^{-1}\right)$ showed most effectual for the control of maize stalk rot caused by Erwinia chrysanthemi pv. zeae. Similarly, Burlakoti and Khatri-Chhetri (2004) also highlighted the foliar spray of streptocycline (100 ppm) was effective for the control of bacterial stalk rot of maize. An application of $75 \%$ captan per 1001 water applied as a soil drench at the base of the plant when the crop is 5-7 weeks old) can check this disease effectively (Payak \& Renfro, 1974). The diseases are known to occur in Nepal, India, Indonesia, Pakistan, Philippines, Thailand and Vietnam. They are observed more commonly if 
there is a period of drought during or shortly after pollination (Subedi, 2015). Agronomically desirable stalk rot-resistant materials are available in Pakistan, India, Mexico and Zimbabwe, where selections against these diseases have been made. The 'stay green' character, in which plants remain green after attaining physiological maturity, has been associated with resistance to certain post-flowering stalk rots (Subedi, 2015). There is evidence of mammalian toxicity where stalks infected with these pathogens.

\section{CONCLUSION}

The result from field monitoring revealed that the maize stalk rot complex was severe in western maize growing belts of Dang while susceptible reaction was marked at Chitwan, Nawalparasi and Surkhet districts. The disease was found mild at Banke district. The higher percent disease control (52.36\%) and yield increase $(40.29 \%)$ were recorded from the plot sprayed with streptocyclin @ $2 \mathrm{~g} \mathrm{~L}^{-1}$ and insecticide (cypermethrin + chloropyrifos @ $2.5 \mathrm{ml} \mathrm{L}^{-1}$ of water during knee height and subsequent spray after 15 days interval as compared to farmers practice. Similarly, the findings from the screening experiment showed that out of 30 genotypes, Rampur Composit, Arun 2, Rampur 34, RamS03F08, TLBRS07F16 and Rampur 24 were found resistant against stalk rot complex with higher yield at Rampur Chitwan.

\section{ACKNOWLEDGEMENTS}

The authors are thankful to the NMRP Coordinator and CIMMYT for financial support in this study. The research team of NMRP, Rampur and CIMMYT- CSISA are gratefully acknowledged for trial management and data recording. All peoples who provided valuable opinions and ideas on this manuscript are also appreciated.

\section{REFERENCES}

Burlakoti, R.R., \& K.C, G.B. (2004). Bacterial diseases of crop plants in Nepal: a review. Journal of Institute of Agriculture and Animal Science, 25, 1-10.

Campbell, C. L., \& Madden, L.V. (1990). Introduction to Plant Disease Epidemiology. John Wiley \& Sons, New York City.

De Leon, C. (1984). Maize Diseases, a Guide for Field Identification, 3rd edn. CIMMYT, EI Batan, Mexico.

Diwakar, M. C., \& Payak, M.M. (1980) Influence of some environmental and host factors on Pythium stalk rot of maize. Indian Phytopathology. 33, 10-15

Diwakar, M.C., \& Payak, M.M. (1975). Germplasm reaction to pythium stalk rot of maize. Indian Phytopathology, 28(4), 548-549.

ICAR. (2012). Inoculation methods and disease rating scales for maize diseases. Revised edn. Directorate of Maize Research. Indian Council of Agricultural Research, Pusa Campus, New Delhi-110012. India. Pp. 20-28.

Ledencan. T., Simic, D., Brkic, I., Jambrovic, A., \& Zdunic, Z. (2003). Resistance of maize inbreds and, their hybrids to Fusarium stalk rot. Czec Journal of Genetics and Plant Breeding, 39, 15-20

Oerke, E.C. (2005). Crop losses to pests. The journal of Agricultural Science, 144, 31-43. 
Payak, M. M., \& Renfro, B.L. (1974). A decade of research on maize diseases: impact on production and its international cooperative outreach. In: Current Trends ill Plant Pathology (Ed. by S. P. Rychaudhuri and J. P. Verma) pp. 160--170, Department of Botany, Lucknow University. India.

Shah, S.M. (1968). Diseases of maize in Nepal. In: Fifth Inter-Asian corn improvement workshop, Department of agriculture and Kesetsart University, Bangkok, Thailand.

Shivankar, S.K., \& Wangikar, P.D. (1993). Effect of different fungicides on the control of gray mildew disease of cotton. Indian Phytopathology, 46 (3), 230-235.

Singh, N., Rajendran, A., Meena, S., \& Mittal, G. (2012). Biochemical response and hostpathogen relation of stalk rot fungi in early stages of maize (Zea mays L.) African Journal of Biotechnology, 11(82), 14837-14843

Subedi, S. (2015). A review on important maize diseases and their management in Nepal. Journal of Maize Research and Development, 1(1), 28-52.

Szoke, C.S., Árendas, T., Racz, F., Pinter, J., Nagy, E., \& Marton, L.C. (2007). Correlation between maize genotypes and the stalk rot caused by maize Fusarium. Acta Agronomy Hungarica, 55(4), 447-452.

Thind, B.S., Randhawa, P.S. \& Soni, P.S. (1984). Chemical control of bacterial stalk rot (Erwinia chrysanthemi pv. Zeae) and leaf stripe (Pseudomonas rubilineans) of maize. Journal of Plant Disease and Protection, 91(4), 424-430.

Wheeler, B.E.J. (1969). An introduction to plant diseases. John Wiley and Sons. Ltd. London. pp. 301. 